\title{
Article
}

\section{An ethnographic study exploring the role of ward-based Advanced Nurse Practitioners in an acute medical setting}

Williamson, Susan, Twelvetree, Timothy, Thompson, Jacqueline and Beaver, Kinta

Available at http://clok.uclan.ac.uk/5577/

Williamson, Susan ORCID: 0000-0002-9635-4473, Twelvetree, Timothy, Thompson, Jacqueline and Beaver, Kinta ORCID: 0000-0002-6552-2323 (2012) An ethnographic study exploring the role of ward-based Advanced Nurse Practitioners in an acute medical setting. Journal of Advanced Nursing, 68 (7). pp. 1579-1588. ISSN 03092402

It is advisable to refer to the publisher's version if you intend to cite from the work. http://dx.doi.org/10.1111/j.1365-2648.2012.05970.x

For more information about UCLan's research in this area go to

http://www.uclan.ac.uk/researchgroups/ and search for <name of research Group>.

For information about Research generally at UCLan please go to http://www.uclan.ac.uk/research/

All outputs in CLoK are protected by Intellectual Property Rights law, including Copyright law. Copyright, IPR and Moral Rights for the works on this site are retained by the individual authors and/or other copyright owners. Terms and conditions for use of this material are defined in the policies page. 


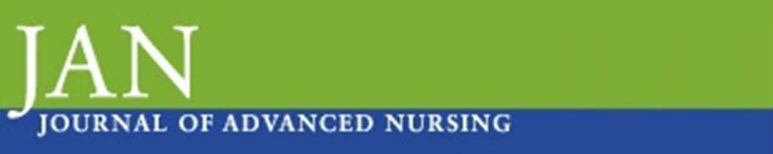

An ethnographic study exploring the role of ward-based advanced nurse practitioners in an acute medical setting

\begin{tabular}{|r|l|}
\hline Journal: & Journal of Advanced Nursing \\
\hline Manuscript ID: & Draft \\
\hline Manuscript Type: & Original Article \\
\hline Keywords: & $\begin{array}{l}\text { Advanced Practice, Ethnography, Skill mix, Clinical Nurse Specialist, } \\
\text { Holistic Care }\end{array}$ \\
\hline Category: & Nursing \\
\hline \multicolumn{2}{|l}{} \\
\hline
\end{tabular}

SCHOLARONE ${ }^{\text {m }}$

Manuscripts 
An ethnographic study exploring the role of ward-based advanced nurse practitioners in an acute medical setting

\begin{abstract}
Aim. This paper is a report of a study that aimed to examine the role of ward based Advanced Nurse Practitioners (ANP) and how they impact on patient care and nursing practice.
\end{abstract}

Background. The international impact of revised doctor/nurse skill mix combined with a focus on improving quality of care whilst reducing costs has altered the pattern of healthcare delivery. The diversity and implementation of advanced nursing practice roles has developed globally over the last decade. However, the role and expectations for ward based ANPs lacks clarity, which may hinder effective contribution to practice.

Methods. This study used an ethnographic approach to explore the ANP role. This included participant observation of five ward based ANPs working in a large teaching hospital in the North West of England during 2009, complemented by interviews with ANPs, 14 ward nurses and five patients. Data were descriptive and broken down into themes, patterns and processes to enable interpretation and explanation.

Results. The overarching concept that ran through the analysis of data was that of the ANP as a lynchpin, using their considerable nursing expertise, networks, and insider knowledge of health care systems not only to facilitate patient care but to develop a pivotal role facilitating nursing and medical practice. Sub-themes included enhancing communication and practice, acting as a role model, facilitating the patients' journey and pioneering the role.

Conclusion. Ward based ANPs are pivotal and necessary for providing quality holistic patient care and their role can be defined as more than junior doctor substitutes.

Key words: advanced nurse practitioners, specialist nurses, skill mix, ethnography, participant observation 


\section{Summary Statement}

\section{What is known}

- The role of Advanced Nurse Practitioners (ANP) in practice is not clearly defined.

- A lack of role definition and management support causes barriers to advanced nursing practice.

- ANPs can undertake some of the role of junior doctors.

\section{What this paper adds}

- The role of the ward based ANP is pivotal to the management and delivery of quality, holistic patient care.

- ANPs are highly valued in a ward setting as they are accessible and provide an interprofessional communication channel, a technical and knowledgeable resource, and continuity of care.

- ANPs perceive inadequacies in their educational preparation.

\section{Implications for Practice and/or Policy}

- Ward based ANPs could improve the response to Early Warning Score (EWS) triggers.

- Ward based ANPs are believed to have a positive impact on length of patient stay and discharge procedures.

- Further work is needed to evaluate the impact of ward based ANPs on admissions to High Dependency or Intensive Care Units (HDU/ICU) 


\section{INTRODUCTION}

The diversity and implementation of advanced nursing practice roles has developed globally over the last decade, often in response to localised initiatives based on revised doctor/nurse skill mix (Ball \& Cox 2003, Bryant-Lukosius et al 2004, Buchan \& Calman et al 2005, Gardner et al 2007). A reduction in junior doctors working hours and more structured supervision requirements for training spurred the development of more specialist and advanced nursing roles, which equipped nurses to take on many procedures and tasks traditionally associated with junior doctors (NHS Executive 1991, Cox 2001). Advanced nursing practice creates enhanced levels of competency with a positive impact on patient care; higher levels of educational preparation impact on patient mortality and patient satisfaction (Aiken et al 1994, Clarke \& Aiken 2003, Rothberg et al 2005). However, there is much confusion about variability in nursing roles, titles, and expectations (Ball \& Cox 2004, Bryant-Lukosius 2004, Laurent et al 2009). To date there has been no investigation of the actual, rather than theoretical or perceived role, of ward based Advanced Nurse Practitioners (ANP) and their potential impact on patient care and nursing practice. This study aimed to address this gap in knowledge, using ethnographic techniques to identify and clarify the role and impact of ward based ANPs in a large teaching hospital in the North West of England.

\section{BACKGROUND}

The term "advanced nursing practice" encompasses many specialist roles within nursing but does not define them and in developed English speaking countries, particularly Australia, Canada, the United Kingdom (UK) and United States (US) has led to the term being used to describe specialist nursing roles with a wide variation in scope of practice and educational qualifications (Aiken et al 1994, Mundinger et al 2000, Ball \& Cox 2003, Bryant-Lukosius et al 2004, Gardner et al 2007, Gardner et al 2008, Pulcini et al 2009). In the US and UK, nurses with advanced practice skills were originally employed in primary care to ease General Practitioners (GP) workload and to provide enhanced primary care services (Brown \& Grimes 1995, Mundinger et al 2000, Horrocks et al 2002). However, it is not clear what added value these highly skilled and trained nurses bring to secondary care. 
Recent studies have attempted to distinguish the often subtle difference between nurses in advanced nursing practice roles such as ANPs and Clinical Nurse Specialists (CNS). A CNS uses advanced nursing skills within a given specialist area while an ANP works across specialism's in much the same way as junior doctors (Gibson \& Bamford 2001,Austin et al 2006, Gardner et al 2007, Mitchell et al 2010). It is interesting that the use of ANPs has been largely confined to specialist areas, as the reduction in junior doctors hours in the UK affected all areas of patient care including general in-patient wards. It is argued that ANPs have not just filled the gap left by a reduction in junior doctors' hours, but also use their expertise to identify and fill gaps in service provision (Ball \& Cox 2004, Gardner et al 2008, Laurent 2009).

Whilst the experience and skills of ANPs can be used to define many of the characteristics of advanced nursing practice, capabilities go beyond these competencies to include high levels of self efficacy, creativity and innovation in complex situations while working effectively as part of multidisciplinary teams (Gardner et al 2007, Gardner et al 2008, Pulcini et al 2009). Additional training and education enable ANPs to perform patient consultations, physical examinations, arrive at a differential diagnosis and prescribe where appropriate. However, on an international level it is evident that hospital based ANPs are often appointed, educated and trained without clear definition of what their employers expect of them (Ball \& Cox 2004, Bryant-Lukosius et al 2004, Buchan \& Calman et al 2005 Garner et al 2008). In spite of studies identifying what the role of ANPs should be and what ANPs are able to do, there is little evidence describing what ANPs actually do to fulfil their role and how this impacts on nursing practice and patient care (Ball \& Cox 2003, Bryant-Lukosius et al 2004,Lloyd-Jones 2005, Gardner et al 2007, Gardner et al 2008, Mitchell et al 2010). This study aimed to examine these issues.

\footnotetext{
AIM

To examine the role of ward based ANPs and how they impact on patient care and nursing practice.
} 
METHOD

\section{Design}

A qualitative ethnographic design was chosen using non-participant observation and semistructured interviews. Ethnography explores people's behaviour in a natural rather than contrived setting in order to interpret and explain behaviour in the context of the rules, roles and expectations of culture (Fetterman 1989, O'Leary 2004, Hammersley \& Atkinson 2007). Observation in a health care setting allows the collection of naturally occurring data and events that are often taken for granted without causing disruption or interruption. It enables the identification of the context in which events happen and examines associations between events and the effectiveness of the actions of the participants (Ritchie \& Lewis 2007). Although individual in-depth interviews are time consuming they complement the observation and are a key part of the interpretation of observed events (Spradley 1980). Interviews allow participants to provide their own interpretation of events and describe personal feelings about experiences within their position in a particular environment.

\section{Participants}

Study participants were recruited in two stages. Initially the practice of five ANP's employed on acute medical wards at a large teaching hospital in the North West of England was observed. Direct observation was considered essential in gaining a full understanding of how ANPs function and communicate in clinical reality as perceived action and actual action may differ. Following the observation interviews were conducted with all five ANP's, a sample of 14 ward nurses, stratified by job title, and five patients.

\section{Data Collection}

\section{Observation}

Prior to commencing the formal observation period, time was spent meeting the ANPs' and shadowing them on the wards to acclimatise them to the study and minimise any distortion that the observation may have. Subsequently each ANP was observed over seven days for between two to three hours each day on different shift patterns until both they and the researcher felt that the observation period had covered an accurate representation of their practice. Detailed notes were made about what and who was being observed, the physical 
setting, interactions and activities, including significant quotes from informal opportunistic interviews with a number of health care professionals including medical consultants, junior doctors, nurses, physiotherapists, occupational therapists and clinical pharmacists. This enabled the observations to be reviewed away from the clinical setting and later during analysis. Notes were taken after each observation period as overt note taking during observation could have been perceived as threatening and disruptive (Hammersley \& Atkinson 2007). Eighty six hours of observation were completed. The observation generated questions, some of which were addressed during informal interviews and others were used to inform interviews.

\section{Interviews}

The five ANPs, 14 ward based nurses and five patients were invited to participate in interviews; time constraints and a change in discharge policy hindered the recruitment of more patients. A diverse range of views were sought from both junior and senior ward nurses. Patients were interviewed at the point of discharge, allowing time to form an opinion about their hospital stay and those who cared for them. Issues that had arisen during observation were integrated into all the interviews (Fontana \& Prokos 2007). The ward environment in which each ANP worked was very different, so individual interviews with ANP's provided an understanding of their feelings about, and interpretation of, their role. They were asked to comment on their perceptions of their role and communication patterns with other health professionals. Ward nurses were asked what they liked and disliked about working with an ANP, the role of an ANP and whether ANP's had an impact on patient care and nursing practice. Patients were asked more general questions about their stay on the ward, how they felt about their nursing care and information given to them about their condition. With consent, all interviews were recorded and transcribed.

\section{Ethical considerations}

Ethical approval was granted by the National Research Ethics Service for England and by the Research and Development Department at the study site. Although ANP's were the focus of observation, other ward staff and patients were present during periods of observation. Therefore, the consent of each ward manager was sought to allow the presence of the researcher in ward areas. All participants, including patients, consented in writing and 
confidentiality was protected through the use of identification numbers and the removal of all identifying features from the data.

\section{Analysis}

Field notes contained the reactions and reflections about the significance of the processes observed. As insights and ideas became apparent they were annotated and formed part of the preliminary analysis of what had been observed. The purpose of the observation was to describe and explain the observed role of the ANP and data were broken down into the concepts, patterns and processes that enabled interpretation and explanations. A second researcher read and annotated the field notes and developed concepts which were then compared and discussed (Spradley 1980, Hammersley \& Atkinson 2007).

Transcripts of interview data were read and open coded independently by two researchers. A framework was developed and the coding re-examined using content analysis. As patterns and themes emerged, categories were identified and comparisons made with the data and preliminary analysis from observations. Any discrepancies were resolved through review and discussion. Reliability was established through the above processes. When analysis was completed the preliminary findings were shown to the ANPs and ward nurse participants to ensure that views and events were reported accurately, thus assisting the validation process (Spradley 1980, Hammersley \& Atkinson 2007).

\section{RESULTS}

\section{Participant characteristics}

ANP participants included two males and three females; all had attained a Masters degree in advanced nursing practice and were experienced nurses having previously worked as senior nurses or CNS's in a variety of clinical settings but not on acute medical wards. All had been in post for about two years and reported that their role was still developing. All 14 ward nurses were female registered nurses, including four ward managers, three ward sisters (registered nurses with a clinical and managerial role), four staff nurses (registered nurses with a clinical role) and three assistant practitioners (nursing assistant with vocational qualifications). Of the five patients, four were female and one was male. 


\section{Key themes}

The overarching concept that ran through the analysis of data was that of the ANP as a lynchpin, ensuring effective communication between those providing, and those receiving, patient care to ensure a quality service. A number of sub-themes emerged from the observation and interview data: enhancing communication and practice, acting as a role model, facilitating the patients' journey and pioneering the role (Figure 1).

\section{ANP as a lynchpin}

A number of roles and skills were observed, with ANPs' facilitating most aspects of patient care. After initial scepticism from medical consultants, they described ANPs as "pivotal", and an invaluable link between medical and nursing teams. ANPs shared responsibility for patients with junior doctors, playing an active part in consultant's ward rounds and, because they were ward based, provided a continuity that junior doctors could not. Their specialist knowledge, technical skills and clinical judgement were respected and their sustained presence on the ward enabled a detailed understanding of each patient's history and circumstances, which was used to expedite early discharge. All ANPs acknowledged that they were an information and communication resource for a diverse range of health care professionals involved in patient care. All grades and types of staff were observed approaching ANPs to ask questions about individual patients' condition, diagnosis and treatment.

"Not only does she help with the doctors jobs or the nurse's jobs, she is kind of a link. You know like a bridge between doctors and nurses." (ID77 Staff Nurse)

"I've had quite a lot of tests.... They do explain to you what they're for, and they do give you printed information... They will all reassure you, but I think the one that will tell you everything about it would be your advanced, because [ANP name]... I think knows more, because he is advanced, ...." (ID 78 patient)

Ward nurses reported that ANP's had a positive impact on nursing practice but considered ANPs to be more closely allied to the medical rather than nursing team. They all agreed that ANPs assisted with nursing work but on the whole felt that they did not actually do any 
"hands on" nursing. However, this view was not shared by ANPs who felt their role enabled them to spend more time practicing nursing.

"I believe I nurse better as an advanced nurse practitioner than I had the opportunity to as a sister in charge of a busy high dependency unit. And what I mean by that is I have the time to sit down alongside a patient and explain things to them, and listen to them. And that's something I really struggled to do in my previous nursing roles, because of other demands on me." (ID60 ANP)

\section{Enhancing communication and practice}

The ANPs were observed to be experienced confident practitioners using subtle and complex communication skills which the younger nurses and doctors had not yet developed. In addition ANPs frequently 'translated' medical instructions for nurses, patients and allied health professionals, to ensure that the significance of planned care was understood. ANPs often returned to patients after a ward round to ensure that they understood what had been said, providing further explanations if necessary using different vocabulary.

"And the fact that [name ANP] understood. He just hit the nail right on the head, and took time, oh he was in here for a long time. But he waited, and kept going until he was satisfied that I was quietly confident and I was reassured enough". (ID78 patient)

Nurses generally found ANPs less intimidating and more approachable than doctors when resolving care issues. Having an ANP available as a resource inspired confidence as nurses felt they always had back up and support. In addition, the ANPs proactive approach meant that they picked up on issues that needed to be addressed to prevent patient deterioration or delayed stay.

"... it's having the back up there, that we know as staff nurses that... what we're supposed to do. But having the ANP there as well she overlooks the obs [observations] on the ward round, and then picks up on anything that was sort of perhaps missed or not undertaken. So it's... Having them there as an added back up really." (ID68 Staff Nurse) 
Role model

In addition to formal teaching and mentorship, ANPs used their technical knowledge and skills to provide informal support and teaching to nurses and junior doctors. This promoted ANPs as role models, which they embraced and appreciated.

"I'm a resource for them.... I'm a member of the team that the majority of nurses don't feel disinhibited in approaching ...So it's what learning opportunities I can then create within that interaction for them". (ID60 ANP)

The ANPs' familiarity with the hospital and their networking skills brought advantages. All the ANPs' were observed supporting and teaching junior doctors when they first started on the wards and were unfamiliar with the organisation. ANPs reported that part of their role was to support and guide junior doctors to enable them to be more efficient whilst they were still familiarising themselves with the hospital systems.

"Well I hope they (junior doctors) see it as helpful. They tell me that they do so... Especially at this time of year, the doctors... But I like that, I like being useful so it's... I like this time of year, 'cos there's always lots of questions, and not just about what's going on with the patients, just general systems within the hospital and that sort of thing... I feel like mother hen gathering them all up and leading them along." (ID66 ANP)

Occasionally it was observed that having an ANP on the ward seemed to reduce the need for ward nurses to use their initiative and develop their skills. ANPs reported that although it was flattering when ward nurses assumed they would always have answers to their questions, it reduced the need for them to use their analytical skills. De-skilling, therefore, was a possibility, particularly on a busy ward where time was limited.

"And I'm so reluctant to say this, because I'm so grateful for everything [ANP]'s done, if anything he might slightly de-skill me, in that I think oh, that's a difficult blood, I'll ask him to take it. Oh, that's a difficult procedure, but [name ANP]'s here. Whereas if he wasn't I would 
be in a position where I would have to try myself. So I don't know if that slightly de-skills me... (ID 74 Ward Sister)

Facilitating the patients journey

ANP's were observed as pro-active rather than reactive in providing and enabling holistic care for patients. They anticipated what would be needed and actively tried to improve the speed at which tests and investigations were expedited and referrals acted upon. As ANPs were accessible, ward nurses were able to utilise their technical skills and knowledge with immediacy rather than waiting for a junior doctor, which speeded the response to patient needs or deterioration.

"I think I have a crucial impact on the care that they receive, because not only am I involved in their medical management and chasing up appropriate investigations and reviewing them daily, I'm also ensuring that from a nursing point of view that the care that they're receiving is appropriate,... and that the nursing staff are aware of warning signs when they're becoming acutely unwell, and who to refer onto..... So I'm not only thinking about their aetiology at the time, and managing that, I'm thinking about their emotional needs and I'm thinking further ahead from that, I'm thinking well when we get to the point of them being medically fit how are they gonna manage at home, what do we need to be doing now in order to get all this process sorted out for that long-term vision if you like"(ID65 ANP).

A rapid response to patient deterioration was highly valued; patients who may otherwise have "triggered" on the early warning score (EWS) system were subject to prompt intervention by an ANP. Nurses reported that ANPs had enhanced credibility; if the ANP asked one of the doctors to come to the ward to see a patient who was triggering on the EWS they would not be ignored, which again enhanced the care that the patients were given.

"ANP can deal with the situation immediately, and her ringing the doctors to come to review the patient, they will come much quicker than they will with the nurses on the ward ringing to...And usually she's done everything anyway in preparation, she's done all the blood tests, she's done the ECG, she's ordered chest X-ray, put a drip up, so all those things have usually 
been done before the doctors actually get to the ward... you're not wasting as much time." (ID72 Ward Manager)

Pioneering the role

ANP's were observed to be consummate professionals but interviews identified a number of challenges they had faced, indicating little clarity around role expectation or scope of practice. They had to overcome initial scepticism from other health professionals, carve out a role and integrate themselves into the medical and nursing teams on the wards where they were based. The ANP's perceived that they were neither part of the medical or nursing team, yet had to meet competing demands and, in some cases, overcome the antagonism of colleagues.

"I was told a couple of months in to the job, that the consultants didn't want an ANP... They didn't understand what it was, but they didn't want one. " (ID 66 ANP)

"The inherent challenge of an advanced nurse practitioner is to meet everybody's competing demands, because everybody's got a view on what you should be doing. Nurse management have their perspective. The medical team will come with their views and so on and so forth. But somewhere along the line you've got to carve out something that's whilst it meets the operational expectations and demands of the trust [hospital], it's also satisfying..." (ID60 ANP)

Many senior nurses expressed initial misgivings about working with an ANP as they expected some degree of role conflict with concern that ward nurses would be de-skilled as ANPs undertook many of the extended roles that senior nurses had previously undertaken. As a result, ANPs had to find a niche in which to develop their role and prove their sceptics wrong. Each role, therefore, was individually developed by each ANP and it was interesting to note that ANPs expressed a lack of knowledge regarding each others role.

ANPs reported that their Masters degree in advanced nursing practice had not adequately prepared them for their clinical role and they were ill prepared in terms of communication, political awareness and leadership skills. However, as one ANP conceded, how can one 
prepare for something that is not defined? When reflecting back to their initial appointment as ANPs, the primary need was to practice at a high level within a clinical reality that could not be prepared for through academic study alone. ANPs placed a high value on the clinical teaching and support provided by consultant physicians.

"Educationally it's a very intense rushed course really and there's a lot of... Well I didn't feel it was enough. We seemed to rush through each system.......And compared to the junior doctors we don't get the background that they get, and yet we're expected to do some of the things that they do. Your nursing background will fill in some of the gaps, but I didn't feel adequately educationally, clinically trained for the role. (ID66 ANP)

\section{DISCUSSION}

The National Health Service (NHS) management in the UK have called for clear role definitions and role expectations as they strive to improve the quality and value of care for NHS patients (DoH 2010, NHS Institute 2011). The findings from this study confirm that ANP roles are not clearly defined and variation in roles and inconsistent expectations can result in possible role conflict, variable acceptance and role overload (Griffin and Melby 2005, Bryant-Lukosius et al 2004). This study noted variability in roles within one study location. This individualisation makes evaluation and comparisons between ANPs challenging and highlights the importance of ethnographic research to highlight similarities and differences in roles, experiences and expectations. However, in this study there were key roles and associated common tasks which situated ANPs as lynchpins in the wards on which they worked. Figure 2 aims to assimilate these roles and tasks to give an overview of role expectation that may be useful for health care providers when developing job descriptions and person specifications for advanced nursing roles. However, it is acknowledged that providing such structured definitions of expectations could raise tensions between the desire to see roles more formally defined and the professional autonomy an ANP role brings.

It could be argued that role uncertainty and a lack of clarity of roles is an inevitable consequence of increasing specialisation in the nursing profession. However, the interpretive nature of new roles and responsibilities in advanced nursing practice, such as 
ordering and interpreting diagnostic tests and prescribing, is important for ANPs in relation to future role development (Lloyd Jones 2005, Griffin and Melby 2006). The ANPs in this study expressed the view that their Masters degree had not adequately prepared them for their clinical role, perceiving that too much emphasis was placed on academic, rather than the practical skills required for their role. It has been reported that nurses in transition to ANP roles are more likely to place priority on development of clinical skills rather than broader skills such as research, audit and supervision (Griffin and Melby 2006, Woods 1998). Griffin and Melby (2006) argued that Masters education should "ensure that ANPs have the clinical and theoretical knowledge that is fundamental to satisfying core concepts of the role" (Griffin and Melby 2006: p299). A systematic review of role development and effective practice in specialist and advanced practice roles in acute hospital settings reported that characteristics required for effective ANP working included confidence, adaptability, negotiating skills, political astuteness and motivation and creativity (Lloyd Jones (2005). However, these were the skills that ANPs in this study reported as missing from Masters courses; skills that they had to learn on the job. However, a vicious circle is evident here; how can an ANP be prepared for a role that has not been clearly defined? Results from this study may assist in providing a definition and framework to help educationalists focus courses to better prepare ANPs for the clinical workplace.

All grades of staff involved in patient care clearly respected and valued the clinical judgement of ward based ANPs. Their knowledge and skills enabled them to work across specialist boundaries in a similar way to junior doctors, yet they were perceived as more useful as a result of their accessibility, approachability and enhanced technical and communication skills. The ward nurses recognised the ANPs role in expediting patient care and acknowledged a perceived speedier reaction to patient deterioration and corresponding reduction in admissions to the Intensive Care Unit (ICU). However, this study did not seek to evaluate the impact on outcomes of the ANP role and we do not have data on whether admission rates to High Dependency Units (HDU) and ICU had reduced since ANPs commenced in post. If this data were available it would be speculative to suggest a causal link between ANP presence and rapid response to EWS triggers and/or reductions in admissions to HDU/ICU. This warrants further investigation as the response to critically ill 
patients relies on nurses recognising signs of deterioration and reporting them appropriately (Clarke \& Aiken 2003, Rothberg et al 2005, NICE 2007).

This study shows that ward based ANPs play a pivotal role in the patients' journey by providing a communication channel between doctors and nurses, between doctors and patients as well as being an information resource. The introduction of ANPs was viewed by many as a way to reduce work pressure on junior doctors. A question remains whether the NHS and the associated professions are able or willing to continue with this development. Despite the favourable view, and central position of ANPs to patient care in England, acute sector medical and nursing budgets are distinct. If the contributions of ANPs to reducing medical workload are not recognised in a financial way within nursing budgets, there could be concerns that the enthusiasm of nurse managers to support these relatively high grade positions is not sustainable and may not be maintained. Especially as this study demonstrates that although nurses attributed advanced nursing skills such as "knowing" the patients, proactive care, developed recognition skills and effective communication to the ANPs they did not acknowledge these skills as "nursing" and viewed ANPs as being more closely aligned to medicine (Benner \& Tanner 1987, Castledine 1991). This study is timely, given the downturn in the global economy and subsequent impact on health care spending.

Further work is needed to assess the impact of ward based ANPs on response to EWS triggers, patient length of stay and the corresponding economic impact on patients and health care systems (Rothberg et al 2005, Clarke \& Aiken 2003,NHS Institute 2011). This study is limited by the small numbers of ANPs and one study site. However, it can be suggested that ward based ANP roles should aim to improve and develop nursing and medical practice for the benefit of patients by challenging traditions that are not evidenced based, enhancing communication between health care professionals and patients, proactively co-ordinating and facilitating patient investigations, diagnoses, and subsequent care and treatment plans, and act as a resource, role model and mentor to nursing and medical colleagues. Clarifying role expectations should improve preparation and efficiency and go some way to eliminating the challenges and confusion surrounding ANPs. 


\section{Conclusion}

This study achieved its aim of identifying the impact of ward based ANPs on nursing practice and patient care and describing their role. Moreover it shows that ward based ANPs use their considerable nursing expertise, networks, and insider knowledge of health care systems not only to facilitate patient care but to develop a pivotal role facilitating nursing and medical practice. In addition this study demonstrates that because of their nursing heritage, increased skills and knowledge, ward based ANPs are more than junior doctor substitutes. 


\section{REFERENCES}

Aiken LH, Smith HL, Lake ET (1994). Lower Medicare mortality among a set of hospitals known for good nursing care. Medical Care 32(8), $771-787$

Austin L, Luker K, Rowland M (2006) Clinical nurse specialists as entrepreneurs: constrained or liberated? Journal of Clinical Nursing 15, 1540-1549

Ball C, Cox CL (2003) Part one: Restoring patients to health-Outcomes and indicators of advanced nursing practice in adult critical care. International journal of nursing practice 9 , 356-367

Ball C, Cox CL (2004) Part two: The core components of legitimate influence and the conditions that constrain or facilitate advanced nursing practice in adult critical care. International Journal of Nursing Practice 10, 10-20

Brown SA, Grimes DE (1995) A meta analysis of nurse practitioners and nurse midwives in primary care. Nursing Research 44, 332-339

Bryant-Lukosius D, Dicenso A, Browne G, Pinelli J (2004). Advanced practice nursing roles: development, implementation and evaluation. Journal of Advanced Nursing 48(5), 519-529

Buchan L, Calman L, (2005) Skill-Mix and Policy Change in the Health Workforce: Nurses in Advanced Roles. OECD Health Working Papers (17)

Clarke P, Aiken LH (2003). Failure to rescue. American Journal of Nursing 103(1), $42-47$

Cox C. Advanced nurse practitioners and physician assistants. What is the difference? Comparing USA and UK. Hospital Medicine 2001; 62: 169-171

Department of Health (2010) Equity and Excellence: Liberating the NHS. TSO London

Gardner G, Chang A, Duffield C (2007) Making nursing work: breaking through the role confusion of advanced practice nursing. Journal of Advanced Nursing 57(4) 382-391

Gardner A, Hase S, Gardner G, Dunn S, Carryer J (2008). From competence to capability: a study of nurse practitioners in clinical practice. Journal of Clinical Nursing 17, 250-258

Griffin M, Melby V (2006) Developing an advanced nurse practitioner service in emergency care: attitudes of nurses and doctors. Journal of Advanced Nursing 56(3) 292-301

Gibson F, Bamford O (2001) Focus group interviews to examine the role and development of the clinical nurse specialist. Journal of Nursing Management 9, 331-342

Fetterman DM (1989). Ethnography: Step by Step. Sage Publications Ltd: California 
Fontana A and Prokos AH (2007) The Interview. From Formal to Postmodern. Walnut Creek, California. Left Coast Press

Hammersley M, Atkinson P (2007). 3'rd Ed. Ethnography: principles in practice. Routledge, New York

Horrocks S, Anderson E, Salisbury C, (2002) Systematic review of whether nurse practitioners working in primary care can provide equivalent care to doctors. British Medical Journal 324, 819-823

http://www.institute.nhs.uk/quality and value/introduction/quality and value.html accessed 17.03.11

International Council of Nurses: International Nurse Practitioner-Advanced Practice Nursing Network, Research Subgroup. (2001) Update: International survey of nurse practitioneradvanced practice nursing roles. Retrieved January 4, 2011 from http://icn-apnetwork.org/

Laurent M, Reeves D, Hermens R, Braspenning J, Grol R, Sibbald B (2009)Substitution of doctors by nurses in primary care (review). The Cochrane Collaboration. John Wiley and Sons. London

Lloyd-Jones M (2005) Role development and effective practice in specialist and advanced practice roles in acute hospital settings: systematic review and meta analysis. Journal of Advanced Nursing 49 (2), 191-209

Mitchell T, Butler-Williams C, Easton K,Ingledew I, Parkin D, Wade S, Warner R (2010) The consultant nurse-expert practitioner and much more. British Journal of Nursing 19 (8) 481488

Mundinger $M$, Kane R, Lenz R, Totten AM, Tsai WY, Cleary PD, Friedewald WT, Siu AL, Shelanski ML (2000) Primary Care outcomes in patients treated by nurse practitioners or physicians: a randomised controlled trial. JAMA 283 (1), 59-68

National Health Service Executive (1991) Junior Doctors: The New deal. Making the Best Use of Skills of Nurses and Midwives, National Health Service executive, London.

National Institute of Clinical Excellence (2007) Acutely III Patients In Hospital. NICE: London

NHS Institute (2008) www.evidence.nhs.uk/qualityandproductivity accessed 23.02.11

O'Leary Z. (2004) The Essential Guide To Doing Research. Sage Publications Ltd: London

Pulcini J, Jelic M, Gul R, Loke AY (2009) An International Survey on Advanced Practice Nursing Education, Practice, and Regulation. Journal of Nursing Scholarship 42, 31-39

Ritchie J and Lewis J (2007) Qualitative Research Practice. A Guide for Social Science Students and Researchers. London. Sage. 
Rothberg MB, Abraham I, Lindenauer PK, Rose DN (2005) Improving nurse-to-patient staffing ratios as a cost effective safety intervention. Medical Care 43(8), $785-791$.

Spradley JP. (1980) Participant Observation. Stamford Connecticut USA Wadsworth, Thomson Learning. 
Figure 1. Key themes and sub-themes

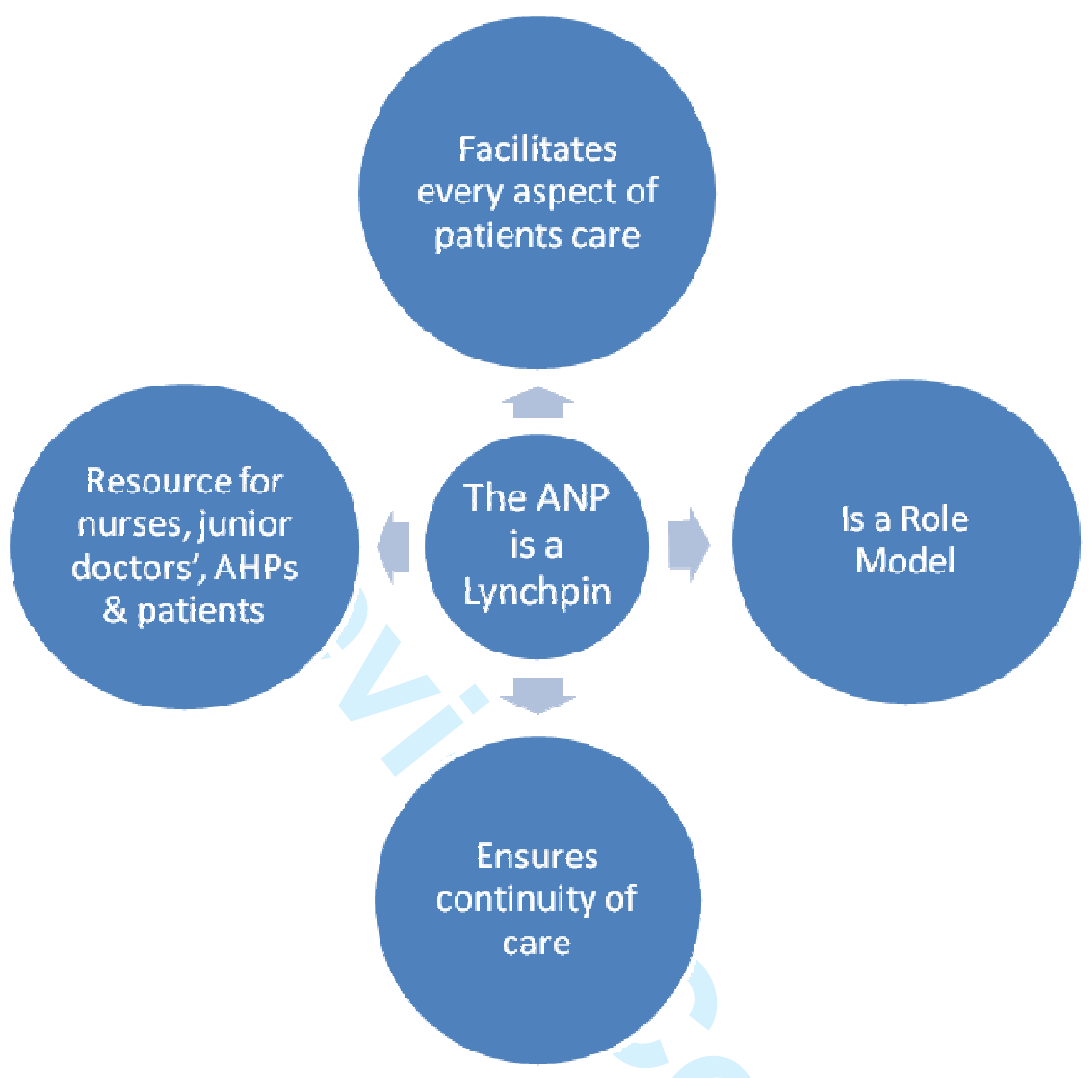

36

37

38

39

40

41

42

43

44

45

46

47

48

49

50

51

52

53

54

55

56

57

58

59

60 
Figure 2. ANP role description

\begin{tabular}{|c|c|}
\hline Role & Tasks \\
\hline Facilitate every aspect of patient's care & 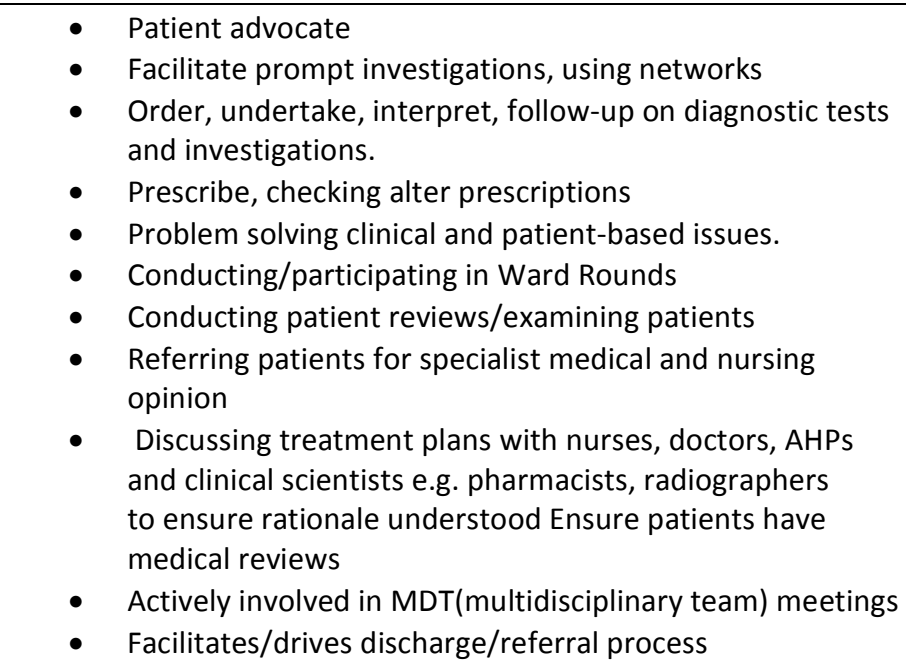 \\
\hline Role model & $\begin{array}{l}\text { Provides advice, knowledge and support to medical, } \\
\text { nursing, and other staff } \\
\text { - Formal and informal teaching of medical and nursing staff } \\
\text { - Literature searching in support of teaching duties or } \\
\text { practice development } \\
\text { - Attend study days/do audit/research/evidenced based } \\
\text { practice/care pathways } \\
\text { - Admin duties/meetings }\end{array}$ \\
\hline Ensures continuity of care & $\begin{array}{ll}\text { - } & \text { Ward based Monday to Friday } \\
\text { - } & \text { Follow-up on ward round actions } \\
\text { - } & \text { Identify anything missed or not undertaken following ward } \\
& \text { round } \\
\text { - } & \text { Driving discharge/referrals } \\
\text { - } & \text { Check/follow up on patients triggering EWS } \\
\text { - } & \text { Liaising with AHPs health and clinical scientists }\end{array}$ \\
\hline $\begin{array}{l}\text { Resource for nurses, junior doctors } \\
\text { AHPs and clinical science staff and } \\
\text { patients }\end{array}$ & $\begin{array}{l}\text { - } \quad \text { Plans care - ensures team approach of all involved } \\
\text { - Prevents delays in treatment/discharge } \\
\text { - Ensures continuity of care } \\
\text { - Ensures provision of holistic care } \\
\text { - Provides cover for doctors } \\
\text { - Share ward round with doctors } \\
\text { - } \quad \text { Translates' medical language into understandable terms } \\
\text { - for nurses, AHPs and patients }\end{array}$ \\
\hline
\end{tabular}

